\title{
EXTENDED MEANING OF CONJUGATE SETS
}

\author{
By W. F. Sheppard.
}

[Received January 2nd, 1921.-Read January 13th, 1921.]

IN connexion with the paper by Prof. Eddington, which precedes this note, I should like to take the opportunity of pointing out that the definition of conjugate sets, in my paper to which he refers, may be made somewhat wider. If $A$ and $B$ are two variable quantities, not necessarily of the same kind, we can denote by $(A ; B)$ some quantity which $(1)$ is determinate when $A$ and $B$ have assigned meanings but is independent of particular values of $A$ and $B$, and (2) satisfies the laws of arithmetic for multiplication, i.e. is such that

$$
(A ; B)=(B ; A), \quad(A ; B+C)=(A ; B)+(A ; C),
$$

and, if $p$ is a constant with regard to $A$ and $B$,

$$
(A ; p B)=p(A ; B) \text {. }
$$

Usually, if $A$ and $B$ relate to a member of a class, $(A ; B)$ will be something depending on the values of $A$ and $B$ for the class as a whole. If, for instance, as in the original paper, $A$ and $B$ were measurements containing errors, $(A ; B)$ might be the mean product of such pairs of errors; or, if $A$ and $B$ were, say, the height and weight of an individual, $(A ; B)$ might be the mean product of the deviations of these from their respective means. A meaning having been given to $(A ; B)$, the definition of conjugate sets, for this meaning of $(A ; B)$, is to be adapted accordingly. Let $A_{\mu} \equiv A_{0}, A_{1}, \ldots, A_{l}$ be a set of $l+1$ variable quantities. Then the conjugate set $A^{\mu} \equiv A^{0}, A^{1}, \ldots, A^{l}$ can be defined either by the condition that $\left(A^{r} ; A_{s}\right)$ is 0 if $r \neq s$ or 1 if $r=s$, or, as in Prof. Eddington's paper, directly by the relation $A^{\nu}=g^{\mu \nu} A_{\mu}$, where $g_{r s} \equiv\left(A_{r} ; A_{s}\right)$.

For finding improved values we require the further condition (3) that $(A ; A)$ is positive unless $A=0$ or a constant, in which case it is $=0$. If $w$ is any linear function of $A_{0}, A_{1}, \ldots, A_{l}$, its improved value, which I will here call $I w$, is defined by the condition that it is the sum of $w$ and a 
linear function of $l-j$ specified $A$ 's (or specified linear functions of the $A$ 's), called auxiliaries, the coefficients in this linear function being chosen so as to make $(I w ; I w)$ a minimum.

With these extensions, Prof. Eddington's methods and results still apply, with the substitution of $(A ; B)$ for the mean product of errors of $A$ and $B$. His notation is adopted in the following supplementary paragraphs; $w, x, y$ denoting definite linear functions of the $A$ 's, and $B_{\mu}, C_{\nu}$, etc. sets of such functions.

(i) We can write $w$ in the form $w=k_{\mu} A_{\mu}$. If also $x=k_{\mu} B_{\mu}$, then $x$ is related to the $B$ 's in the same way that $w$ is related to the $A$ 's. We can express this by saying that $w / A_{\mu}=x / B_{\mu}$; it being understood that a Greek letter in a denominator is what Prof. Eddington elsewhere calls a "dummy," i.e. that the quantity in which it occurs is one of the factors of an "inner product" such as $k_{\mu} A_{\mu}$. We may also have relations such as $A_{\mu} / B_{\nu}=C_{\mu} / D_{\nu}$ or $A_{\mu} / B_{\nu}=E_{\nu} / F_{\mu}$; in this latter case $A_{\mu} F_{\mu}=B_{\nu} E_{\nu}$. These relations can be inverted; e.g. if $A_{\mu} / B_{\nu}=C_{\mu} / D_{\nu}$, then $B_{\nu} / A_{\mu}=D_{\nu} / C_{\mu}$.

(ii) The second and third of the conditions stated under (2) of the first paragraph of this note may be combined in the form

$$
\left(y ; k_{\mu} A_{\mu}\right)=k_{\mu}\left(y ; A_{\mu}\right) \text {. }
$$

If we write $w \equiv k_{\mu} A_{\mu}$, so that $k_{\mu}=w / A_{\mu}$, this becomes

$$
w / A_{\mu}=(y ; w) /\left(y ; A_{\mu}\right)
$$

(iii) If we define a conjugate set in the first of the two ways mentioned in the first paragraph, we easily obtain

or

$$
w=\left(w ; A^{\mu}\right) A_{\mu}=\left(w ; A_{\mu}\right) A^{\mu},
$$

Hence

$$
\begin{gathered}
w / A_{\mu}=\left(w ; A^{\mu}\right), \quad w / A^{\mu}=\left(w ; A_{\mu}\right) . \\
B_{\nu}=\left(B_{\nu} ; A^{\mu}\right) A_{\mu}=\left(B_{\nu} ; A_{\mu}\right) \dot{A}^{\mu} .
\end{gathered}
$$

(iv) From $w=\left(w ; A^{\mu}\right) A_{\mu}$ it follows that

$$
(w ; y)=\left(w ; A^{\mu}\right)\left(A_{\mu} ; y\right),
$$

since $\left(w ; A^{\mu}\right)$ is a constant as regards $w$ and $y$. Hence

$$
\left(B_{\nu} ; C_{\rho}\right)=\left(B_{\nu} ; A^{\mu}\right)\left(A_{\mu} ; C_{\rho}\right) .
$$

(v) For two related sets, and their conjugates, we have four relations 
of the form

$$
\frac{A^{\mu}}{B_{\nu}}=\frac{B^{\nu}}{A_{\mu}}=\left(A^{\mu} ; B^{\nu}\right)=\frac{Q}{A_{\mu} B_{\nu}},
$$

where $Q \equiv A_{\mu} A^{\mu}$. This inner product $Q$ is invariant for any particular original set, and may be expressed either as a quadratic form of the menbers of a related set, the coefficients being the $(;)$ of members of its conjugate set-e.g.

$$
Q=\left(C^{0} ; C^{0}\right) C_{0} C_{0}+2\left(C^{0} ; C^{1}\right) C_{0} C_{1}+\left(C^{1} ; C^{1}\right) C_{1} C_{1}+\ldots
$$

-or in the more general form $\left(C^{\mu} ; D^{\nu}\right) C_{\mu} D_{\nu}$.

(vi) Relations similar to those mentioned above may hold between sets of coefficients or of other constants. If, for instance, $A_{\mu}$ and $B_{\nu}$ are related in a specified way, and if $w$ is a linear function of $B_{\nu}$ which we want to express in terms of $A^{\mu}$, then, if we write

$$
v=p_{\mu} A^{\mu}, \quad q_{\nu} \equiv\left(w ; B_{v}\right),
$$

we have $p_{\mu}=v 0 / A^{\mu}=\left(2 v ; A_{\mu}\right)=\left(w ; A_{\mu} / B_{v} . B_{v}\right)=A_{\mu} / B_{v} . q_{v}$,

so that

$$
p_{\mu} / q_{\nu}=A_{\mu} / B_{\nu} .
$$

'Thus the $p$ 's are related to the $q$ 's in the same way that the $A$ 's are related to the $B$ 's.

(vii) The relation between improved values and original values is such that

$$
I k_{\mu} A_{\mu}=k_{\mu} I A_{\mu}, \quad \text { or } \quad I w / I A_{\mu}=w / A_{\mu} .
$$

It may be noted that a set of $l+1$ improved values has no conjugate set, as the $l+1$ values are not independent but are connected by $l-j$ relations.

(viii) The problem of finding improved values may be expressed as a problem of finding parts of two conjugate sets in succession. Take $A_{\mu} \equiv A_{F} \& A_{R}$, where $A_{F}$ are $j+1$ quantities whose improved values $I A_{F}$ are required, and $A_{R}$ are the $l-j$ auxiliaries. Let the set conjugate to $A_{F} \& A_{R}$ be $A^{F} \& A^{R}$ : and let the set conjugate to $A^{F} \& A_{R}$ be $B_{F} \& B^{R}$. Then $B_{F}=I A_{F}$. For finding either or both of $A^{F}$ and $B_{F}$ we may replace $A_{R}$ by any $l-j$ linear functions of $A_{R}$, and may add to members of $A_{F}$ any linear functions of $A_{R}$. 